\title{
Organisation d'une comparaison inter-laboratoires dans le domaine des essais sur les antiseptiques et les désinfectants
}

\author{
Boris GEYNET \\ Centre Technologique Méditerranéen de Métrologie (CT2M) Centre des Creusets 13250 SAINT-CHAMAS
}

\begin{abstract}
CT2M (Mediterranean Metrology Technology Centre) has been requested by several ISO/CEI 17025 accredited laboratories to organize an interlaboratory comparison in the field of antiseptics and disinfectants testing according to

NF EN 1275 et NF EN 1276 standards. Participating in proficiency testing is a requirement for accredited laboratories but results from precedent European campaigns could not be used by participants. The objectives of this interlaboratory comparison are to assess the laboratories ability to perform these tests, to ensure the quality of results and to estimate the measurements uncertainties. The test organisms are Candida albicans and Staphylococcus aureus according to NF EN 1275 and NF EN 1276 standards respectively. Our innovative ICL protocol takes place in two steps. During the first step, the tested concentrations of disinfectant are not imposed in order to obtain a reference value of two measurands (bactericidal activity and yeasticidal activity), leaving the participating laboratories apply their own routine procedure. During the second step, the concentrations of the disinfectant are imposed in order to better compare laboratories with each other. Successive dilutions of the initial solution / disinfectant neutralized mixture provide a quantitative result for all laboratories and let a statistical exploitation of the reduction rate R according to ISO 13528. This paper will explain the protocol proposed for this interlaboratory comparison, and the statistical treatment to establish participants' performances scores and measurement uncertainty.
\end{abstract}

\section{Introduction}

Présent dans les domaines de la qualité et de la métrologie depuis plus de 20 ans, le CT2M a été sollicité par plusieurs laboratoires accrédités ISO/CEI 17025 pour organiser une comparaison inter-laboratoires dans le domaine des essais sur les antiseptiques et désinfectants chimiques selon les normes NF EN 1275 et NF EN 1276. Le CT2M avait déjà organisé en 2004 une intercomparaison dans le domaine de la microbiologie selon la norme EN 1276.

Avec la collaboration de l'IRM (Institut de Recherche Microbiologique) et d'ANIOS, une campagne de comparaison inter-laboratoires a été lancée sur les essais quantitatifs de suspension pour l'évaluation de l'activité bactéricide et levuricide des antiseptiques et des désinfectants chimiques selon les protocoles européens normalisés EN 1275 et EN 1276.

Cette démarche innovante dans le domaine de la microbiologie permet :

- d'évaluer l'aptitude des laboratoires à réaliser ces essais,
- d'assurer la qualité des résultats,

- de répondre aux exigences normatives (Norme ISO 17025, Norme ISO 10012 (§ 8.3.2)) et d'accréditation,

- de participer à l'estimation des incertitudes de mesure sur les essais concernées.

Cette inter-comparaison s'adresse à des laboratoires :

- accrédités, en cours d'accréditation ou sans accréditation,

- français, européens ou internationaux,

- de différents domaines d'activité (recherche publique ou privée, industrie, services,...).

18 laboratoires européens (français, anglais, allemands, autrichiens et espagnols) ont participé à cet essai d'aptitude. Au moment de l'écriture de cet article, le rapport final de cet essai d'aptitude n'a pas encore été transmis aux laboratoires participants. Pour des raisons de confidentialité, cet article ne dévoile donc pas les 
résultats de la campagne mais décrit simplement le protocole mis en place comportant les dispositions organisationnelles, qualité et techniques pour cette campagne d'intercomparaisons 2015 qui sera renouvelée annuellement. Les résultats détaillés de la campagne seront présentés au Congrès International de Métrologie en septembre 2015 sous forme de conférence affichée.

\section{Organisation de l'essai d'aptitude}

Le désinfectant à tester choisi pour l'essai d'aptitude est une solution commerciale de glutaraldéhyde à $50 \%$ en conditionnement d' 1 litre qui a été distribuée aux 18 laboratoires participants en février 2015. Avant essai, le désinfectant devait être conservé par les participants au frais et à l'abri de la lumière selon les recommandations du fabricant.

Avant la campagne, des essais préliminaires ont été réalisés par deux laboratoires experts du domaine afin de déterminer approximativement l'activité bactéricide et l'activité levuricide attendues. L'objectif de ces essais était par ailleurs de valider le neutralisant (Polysorbate 80, $30 \mathrm{~g} / 1+$ Lécithine, $3 \mathrm{~g} / 1+$ L-histidine, $1 \mathrm{~g} / \mathrm{l})$ qui serait ensuite imposé aux participants afin de limiter la variabilité des résultats due à ce paramètre d'influence.

\section{Première étape de l'essai d'aptitude}

La première étape consiste à réaliser des essais sur le désinfectant défini en respectant les exigences des normes EN 1275 et EN 1276, dans les mêmes conditions que pour un échantillon de routine.

Compte tenu de la durée des analyses, un seul essai selon la norme adaptée a été demandé pour chaque souche. Pour le dénombrement, le prélèvement d'un échantillon de chaque dilution en double est réalisé (duplicats pour chaque concentration du désinfectant testée), comme indiqué dans les protocoles normalisés.

Pour cette phase, les concentrations testées du désinfectant n'ont pas été imposées ; les études préliminaires nous ont tout de même permis de fixer une plage de concentrations à tester comprises entre 0,05 et 1,75 volume/volume afin que les laboratoires ne s'égarent pas vers des concentrations trop éloignées des résultats attendus. Le pas maximal entre les différentes concentrations testées a été fixé pour des raisons liées à l'exploitation des résultats, en effet un pas trop important limiterait l'intérêt de l'exploitation statistique car la variabilité entre les laboratoires ne serait pas représentative. Le rapport entre 2 concentrations consécutives du désinfectant testées est donc laissé au libre choix du laboratoire mais doit rester inférieur ou égal à 2

(pas de raison 2 maximum).

Le délai laissé aux laboratoires pour la réalisation de ces premiers essais est de deux mois après la réception du désinfectant. Un fichier résultats sous Excel a été mis à disposition des participants afin que tous les laboratoires renseignent leurs résultats sous la même forme et ainsi éviter les erreurs d'interprétation par l'organisateur.

Cette phase 1 permet d'exploiter statistiquement les résultats sur les mesurandes suivants : - activité bactéricide, - activité levuricide.

Le traitement des résultats de cette première phase se fait selon les principes statistiques de l'ISO 13528. La valeur de référence ainsi que l'écart-type de l'essai d'aptitude sont déterminés par une analyse robuste et les scores de performances des participants sont calculés de manière classique. L'incertitude sur les deux mesurandes peut être déterminée en appliquant les principes de la série des normes ISO 5725. 


\section{Deuxième étape de l'essai d'aptitude}

La phase 2 débute qu'après réception des résultats de l'ensemble des participants par le CT2M. Dans cette étape, deux concentrations du désinfectant à tester sont imposées. Celles-ci sont définies selon les résultats de la phase 1, afin que la majorité des résultats soient exploitables.

Comme pour la phase 1, un seul essai selon la norme adaptée est demandé pour chaque concentration du désinfectant et pour chaque souche. Ces essais sont à réaliser dans un délai maximum de deux mois après le lancement de la phase par le CT2M. Les résultats de la phase 2 pour cette première campagne ont été reçus en Juin 2015.

L'objectif de cette phase est d'exploiter quantitativement le taux de réduction $\mathrm{R}$ déterminé par les laboratoires pour chacune des deux concentrations imposées du désinfectant, l'interprétation statistique des résultats se fera alors sur ce mesurande (résultat quantitatif sur une échelle continue).

Afin d'obtenir un résultat quantitatif pour chaque participant et pour chacune des deux concentrations testées du désinfectant, le protocole suivant a été mis en place :

- dans le cas où, pour ces concentrations du produit, un dénombrement compris entre 14 et 330 UFC est obtenu pour les 2 duplicats, le laboratoire rend ce résultat et ne procède à aucune dilution.

- Dans le cas où le dénombrement est inférieur à 14 UFC, le laboratoire rend le résultat et ne procède à aucune dilution.

- Dans le cas où le dénombrement est supérieur à 330 UFC, le laboratoire procède à des dilutions successives du mélange d'essai neutralisé jusqu'à l'obtention d'un dénombrement compris entre 14 et 330 UFC (pour chacun des deux duplicats).
Pour chacune des deux concentrations du désinfectant testé, le résultat (taux de réduction $\mathrm{R}$ ) est indiqué dans le fichier de résultats fourni par le CT2M.

Les dilutions successives du mélange d'essai neutralisé sont réalisées de la façon suivante :

- $\quad 1 / 10$ du mélange d'essai neutralisé

- 1/100 du mélange d'essai neutralisé

- $\quad 1 / 1000$ du mélange d'essai neutralisé,

- etc...

Le facteur de dilution $\tau$ est pris en compte dans le calcul de la concentration du mélange et donc dans la détermination du taux de réduction $\mathrm{R}$ pour les deux concentrations de désinfectants testées.

Cette deuxième phase de l'essai d'aptitude est très importante car elle permet de comparer les résultats des laboratoires sur le mesurande qui est le taux de réduction $\mathrm{R}$ pour des concentrations de désinfectant imposées. Le résultat est alors quantitatif sur un domaine continu, ce qui n'est pas le cas pour les résultats de la phase 1 (activité bactéricide et activité levuricide) qui sont quantitatifs mais avec des valeurs discrètes choisies par les participants selon leur mode opératoire de routine.

Le traitement des résultats de cette deuxième phase se fait, comme pour la première étape, selon les principes statistiques de l'ISO 13528. La valeur de référence ainsi que l'écart-type de l'essai d'aptitude sont déterminés par une analyse robuste et les scores de performances des participants sont calculés de manière classique. L'incertitude sur le taux de réduction $\mathrm{R}$ peut être déterminée à partir de l'écart-type interlaboratoires, selon les principes de la série des normes ISO 5725.

\section{Conclusion}

L'organisation d'une comparaison interlaboratoires dans le domaine des essais sur les antiseptiques et les désinfectants selon les normes EN 1275 et EN 1276 est une expérience nouvelle et qui répond à un réel besoin pour les laboratoires 
travaillant dans le domaine. La complexité des analyses concernées a imposé la réalisation d'études préliminaires sur le produit soumis à essai et le protocole a dû être adapté afin de garantir des résultats exploitables par le CT2M. La structure de cet essai d'aptitude en 2 phases permet l'exploitation de plusieurs mesurandes, à savoir l'activité bactéricide, l'activité levuricide, mais également le taux de réduction $\mathrm{R}$ sur des concentrations de désinfectant imposées. Cette campagne sera renouvelée annuellement à la demande des laboratoires afin d'évaluer la fiabilité de leurs résultats et de répondre aux exigences de l'accréditation selon l'ISO 17025. 